\section{A novel prion protein variant in a patient with semantic dementia}

Prion diseases are a group of fatal neurodegenerative diseases that can be sporadic, inherited or acquired. Inherited prion diseases are caused by mutations in the prion protein gene, PRNP, usually single nucleotide substitutions or structural variants of an octapeptide repeat encoding region. Although the classical presentation of sporadic Creutzfeldt Jakob disease (CJD) is rapidly progressive ataxia, myoclonus and cognitive decline, the presentation of genetic cases is variable and decline can be much slower.

Prion diseases can mimic many neurodegenerative diseases. Genetic dementias are pleiotropic, with similar clinical syndromes being caused by mutations in different genes; additionally, mutations in a single gene may cause diverse clinical phenotypes. Due to the diagnostic difficulties arising from this clinical and genetic heterogeneity, next-generation sequencing technologies including gene panels and exome sequencing are helpful in elucidating genetic causes of dementia. ${ }^{1}$

Here we report a novel variant in PRNP in a patient diagnosed with semantic dementia, a variant of frontotemporal dementia (FTD). Patients with semantic dementia develop progressive loss of semantic knowledge resulting in significant early language impairment, with subsequent wider cognitive impairment and behavioural problems. MRI appearances are characteristic with focal asymmetric anteroinferior temporal lobe atrophy. The predominant histopathological finding is accumulation of Tar DNA-binding protein 43 (TDP-43) deposits in the temporal and frontal lobes, although other pathologies, including Alzheimer's disease, are occasionally observed. ${ }^{2}$ A Mendelian genetic aetiology is rarely documented.

A woman in her seventh decade presented with a 1-year history of rapidly progressive language problems and behavioural change. She had particular difficulty naming objects and suffered from impaired comprehension, but had preserved recognition of faces and episodic memory. Over the same time period her personality changed, becoming disinhibited and exhibiting obsessional behaviour. She also struggled with cooking but not with self-care or housework. She had no significant surgical or medical history, apart from a 5 -year history of tinnitus and deafness, and took no regular medications.

A family history of dementia was known: her mother developed dementia of uncertain aetiology in her seventh decade, which progressed steadily with a total duration of 10 years. Her father died in his eighth decade of a myocardial infarction. There was no other relevant family history. 
Physical examination revealed bilateral sensorineural hearing loss, globally brisk tendon reflexes, positive pout reflex and mild bilateral limb apraxia, but was otherwise unremarkable. She scored 23/30 on Mini-Mental State Examination (MMSE). On formal neuropsychometric testing, she obtained a verbal IQ of 66 and performance IQ of 78. Testing of specific cognitive domains revealed significantly impaired single word comprehension (Peabody Picture Vocabulary Test) and anomia (Oldfield Naming Test), as well as a surface dyslexia and dysgraphia, consistent with an impairment of verbal semantic knowledge. Other cognitive domains, including calculation, non-verbal reasoning (Raven's Coloured Matrices), episodic memory (Camden Pictorial Recognition Memory Test) and visuospatial skills, were intact.

Dementia screening bloods and cerebrospinal fluid analysis (undertaken prior to the availability of $\mathrm{A} \beta 42$ and tau measurements) were normal. An MRI scan was reported as showing scattered white matter lesions in both cerebral hemispheres, presumed secondary to mild small vessel disease, with evidence of generalised brain atrophy, although the scan was unavailable for review.

A diagnosis of semantic dementia was made based on the history and pattern of her cognitive deficits.

She was next reviewed 5 years later when she had deteriorated significantly with very limited comprehension and frequent use of jargon words, as well as presenting with visual agnosia. Her behaviour had deteriorated, becoming severely apathetic, losing interest in all of her previous hobbies, increasingly obsessive, particularly with time-keeping, markedly disinhibited, and developing a sweet tooth. She could no longer do housework and struggled with personal activities of daily living.

The patient was subsequently lost to follow-up and died 10 years after onset without being examined at postmortem.

Twenty years after presentation, a DNA sample was tested using the Medical Research Council (MRC) Dementia Gene Panel, a custom next-generation sequencing technology covering 17 dementia genes, including common FTD-associated genes such as MAPT and $G R N .^{1}$ Samples were tested separately for C9orf72 expansions and PRNP octapeptide repeat insertions/deletions. Results were analysed for known and novel variants and confirmed by Sanger sequencing.

We discovered a novel PRNP variant (R156C, g.4680332C > T), which is absent from 141362 unrelated individuals' exome and whole-genome sequences collated by the Exome Aggregation Consortium (http://gnomad.broadinstitute.org/). The patient was homozygous for methionine at codon 129 (129MM). No other pathogenic mutations or risk factors for neurodegeneration were

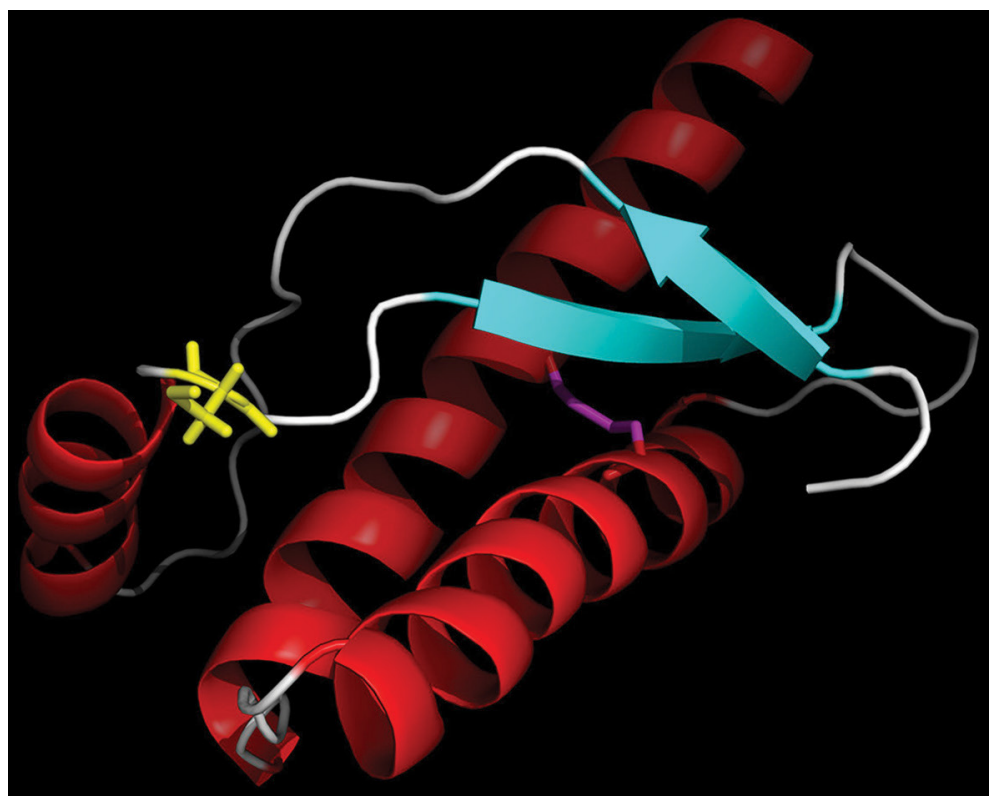

Figure 1 Ribbon representation of the structure of the folded domain of the human prion protein, ${ }^{3}$ with $\alpha$-helices coloured red, $\beta$-strands cyan and loops white. The mutated cysteine side chain of residue 156 is shown in yellow. The disulphide bond linking the side chains of the other cysteines in the protein (Residues 179 and 214) is shown in magenta. This figure was prepared using PyMOL (Schrödinger). identified. The variant results in the replacement of a large, positively charged arginine residue, located immediately after the first $\alpha$-helix of the prion protein $\left(\operatorname{Pr} \mathrm{P}^{\mathrm{C}}\right)$, with a smaller polar, uncharged cysteine (figure 1). As this amino acid is located on the $\operatorname{PrP}^{\mathrm{C}}$ surface, it is unlikely to cause a gross conformational change in $\operatorname{PrP}^{\mathrm{C}}$. However, cysteines may form intermolecular or intramolecular covalent disulphide bonds. Indeed, the two wild-type cysteines present in $\operatorname{PrP}^{\mathrm{C}}$ form a disulphide bond, which is essential to the fold stability of the protein. ${ }^{3}$ We hypothesise that the introduction of an additional cysteine might disrupt the normal $\mathrm{PrP}^{\mathrm{C}}$ disulphide bond or result in the formation of intermolecular disulphide bonds, as observed previously. ${ }^{4} \mathrm{~A}$ similar arginine to cysteine mutation, R208C, has been found in a single case with CJD. ${ }^{5}$ A few other mutations have been reported in the region between first and second $\alpha$-helices including Q160X, Y163X, D167G and D167N, some of which follow a very atypical clinical course. ${ }^{6}$ No mutations have been reported at position 156, but in silico modelling (Polyphen, SIFT, FATHMM, MutationAssessor, MutationTaster) suggests that this variant is likely to be pathogenic. However, Minikel et al ${ }^{7}$ demonstrated that the frequency of PRNP missense and truncating variants in the general population is incompatible with the incidence of prion diseases. Novel variants thus need to be assessed with caution regarding their pathogenicity in the absence of segregation or functional data despite their rarity.

We report a novel missense mutation of $P R N P$ in a patient with semantic dementia, which would at present be classified as likely pathogenic. Patients with semantic dementia would not normally be recommended for genetic studies, particularly of PRNP. The use of next-generation sequencing technologies will be increasingly important in elucidating the causes of pleiotropic conditions such as familial dementia.

\section{Joanna Kenny, ${ }^{1,2}$ Ione Woollacott, ${ }^{3}$ Carolin Koriath, ${ }^{3}$ Laszlo Hosszu, ${ }^{1}$ Gary Adamson, ${ }_{1}^{1}$ Peter Rudge, ${ }^{2}$ Martin N Rossor, ${ }^{3}$ John Collinge, ${ }^{1,2}$ Jonathan D Rohrer, ${ }^{3}$ Simon Mead ${ }^{1,2}$}

${ }^{1}$ MRC Prion Unit, Department of Neurodegenerative Disease, UCL Institute of Neurology, London, UK ${ }^{2}$ NHS National Prion Clinic, National Hospital for Neurology and Neurosurgery, University College London Hospitals NHS Foundation Trust, London, UK ${ }^{3}$ Department of Neurodegenerative Disease, UCL Institute of Neurology, London, UK

Correspondence to Professor Simon Mead, MRC Prion Unit, Department of Neurodegenerative Disease, 
UCL Institute of Neurology, London WC1N 3BG; s. mead@prion.ucl.ac.uk

Acknowledgements The authors would like to thank Professor Elizabeth Warrington for discussion of the case. This study was funded by the Medical Research Council and the NIHR Biomedical Research Centre at UCLH NHS Foundation Trust.

Contributors JK analysed the clinical notes and jointly with CK prepared the manuscript. IW contributed to the clinical data. LH performed the analysis of the crystal structure. GA performed the genetic analysis. PR assessed the patient clinically in their lifetime. MNR and JDR oversaw the clinical assessment. JC and SM oversaw the lab work, genetic analysis and interpretation.

Funding This work was funded by the Medical Research Council (UK) with some support from the National Institute of Health Research's Biomedical Research Centre at University College London Hospitals NHS Foundation Trust. Part of this work was supported by the NIHR Queen Square Dementia Biomedical Research Unit and the Leonard Wolfson Foundation. MNR is an NIHR Senior Investigator. IW is funded by a Clinical Research Training Fellowship from the Medical Research Council.

Competing interests None declared.

Ethics approval UCL Ethics Committee.

Provenance and peer review Not commissioned; externally peer reviewed.

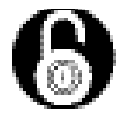

\section{OPEN ACCESS}

Open Access This is an Open Access article distributed in accordance with the terms of the Creative Commons Attribution (CC BY 4.0) license, which permits others to distribute, remix, adapt and build upon this work, for commercial use, provided the original work is properly cited. See: http://creativecommons.org/licenses/by/4.0/

(c) Article author(s) (or their employer(s) unless otherwise stated in the text of the article) 2017. All rights reserved. No commercial use is permitted unless otherwise expressly granted.

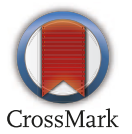

To cite Kenny J, Woollacott I, Koriath C, et al. J Neurol Neurosurg Psychiatry 2017;88:890-892.

Received 16 January 2017

Revised 13 March 2017

Accepted 20 March 2017

Published Online First 1 June 2017

J Neurol Neurosurg Psychiatry 2017;88:890-892.

doi:10.1136/jnnp-2017-315577

\section{REFERENCES}

1 Beck J, Pittman A, Adamson G, et al. Validation of nextgeneration sequencing technologies in genetic diagnosis of dementia. Neurobiol Aging 2014;35:261-5.

2 Hodges JR, Patterson K. Semantic dementia: a unique clinicopathological syndrome. Lancet Neurol 2007;6:1004-14.

3 Hosszu LL, Wells MA, Jackson GS, et al. Definable equilibrium states in the folding of human prion protein. Biochemistry 2005; 44:16649-57.

4 Knaus KJ, Morillas M, Swietnicki W, et al. Crystal structure of the human prion protein reveals a mechanism for oligomerization. Nat Struct Biol 2001;8:770-4.

5 Zheng L, Longfei J, Jing Y, et al. PRNP mutations in a series of apparently sporadic neurodegenerative dementias in China. Am J Med Genet B Neuropsychiatr Genet 2008;147B:938-44.

6 Mead S, Gandhi S, Beck J, et al. A novel prion disease associated with diarrhea and autonomic neuropathy. $N$ Engl J Med 2013;369:1904-14.

7 Minikel EV, Vallabh SM, Lek M, et al. Quantifying prion disease penetrance using large population control cohorts. Sci Trans/ Med 2016;8:322ra9. 